\title{
Lagging-strand origins of the promiscuous plasmid pMV158: physical and functional characterization
}

\author{
M. Gabriela Kramer, Gloria del Solar and Manuel Espinosa
}

Author for correspondence: Manuel Espinosa. Tel: +34 15611800 (ext. 4209). Fax: +34 15627518.
e-mail: CIBME13@CC.CSIC.ES

Centro de Investigaciones Biológicas, CSIC, Velázquez, 144, E-28006 Madrid, Spain

\begin{abstract}
The streptococcal plasmid pMV158 replicates by a rolling circle mechanism, which involves the generation of single-stranded plasmid DNA intermediates. This plasmid has the unique feature of having two lagging-strand origins of replication. One of these origins, termed ssoU, is functional in Streptococcus pneumoniae and in Bacillus subtilis in an orientation-dependent manner. The other origin, SSOA, is only functional in the former host. RNA polymerase seems to be involved in the initiation of the conversion of single- to doublestranded plasmid DNA from both SsoA and ssoU. Mutational and deletion analyses have allowed us to define $550 A$ as being within a highly structured, non-coding 199 bp region. Within this region, two elements which are conserved in several rolling-circle replicating plasmids are located, the recombination site $\mathrm{RS}_{\mathrm{B}}$ and $\mathrm{a} 6$ base consensus sequence. Both elements may play a role in the conversion of single- to double-stranded plasmid DNA.
\end{abstract}

Keywords: Lagging-strand origins, RNA polymerase, rolling-circle replication, singlestranded DNA

\section{INTRODUCTION}

Circular, double-stranded (ds) bacterial plasmids replicate by mechanisms involving the generation of theta-shaped, D-looped, or sigma-shaped DNA intermediates. The last mechanism is used by many small multicopy plasmids, and is termed asymmetric rolling-circle (RC) replication because of the uncoupling of leading- and lagging-strand syntheses (reviewed by Novick, 1989; Gruss \& Ehrlich, 1989; del Solar et al., 1993a). Initiation of RC-replication is mediated by the plasmid-encoded Rep protein which introduces a strand- and site-specific nick on supercoiled plasmid DNA at the plasmid double-strand origin $(d s o)$. Rep activity leaves a $3^{\prime}-\mathrm{OH}$ end which is extended by host proteins while the parental strand is being displaced, and replication proceeds around the whole plasmid, until the entire dso is synthesized. As a consequence of the termination reactions, the parental $(+)$ strand is released as a single-stranded (ss) DNA intermediate (te Riele $e t$ al., 1986), which is the hallmark of RC-replicating plasmids (Gruss \& Ehrlich, 1989). The last stage of RC-replication involves the conversion of ss- to ds-plasmid DNA by the synthesis of the lagging strand (Gruss et al., 1987; del Solar et al., 1987). This synthesis initiates in a plasmid

Abbreviation: $\mathrm{RC}$, rolling circle. region distant from the $d s o$, the single-strand origin, sso (Gruss et al., 1987; del Solar et al., 1987, 1993b). RCreplicating plasmids are constructed like cassettes of genetic information (Projan \& Novick, 1988), one of the modules being sso (del Solar et al., 1993a). Based on sequence homologies, various types of sso have been described (Novick, 1989), including sso $A$, which is present in plasmids pT181, pLS1 and pC194 (Novick, 1989), and ssoU, which is characteristic of plasmid pUB110 (Boe et al., 1989; Birch \& Khan, 1992). A more complex situation is found in the streptococcal plasmid pMV158 (from which plasmid pLS1 is derived; Lacks et al., 1986; Priebe \& Lacks, 1989), because it bears both types of sso (van der Lelie et al., 1989). This feature may provide the plasmid with greater flexibility for adapting itself to the different hosts in which it replicates, but poses the question of whether there is preferential lagging-strand origin utilization within a given host.

In general, the sso regions are located within highly structured non-coding regions (Novick, 1989), perhaps with the exception of plasmid pR (Gigliani et al., 1993). The host machinery recognizes the sso in an orientationdependent manner (Birch \& Khan, 1992; del Solar et al., 1993b). These findings suggest that unpaired sequences, within the secondary structures that constitute the sso, are important for its recognition by host proteins involved in 
$\mathrm{ss} \rightarrow \mathrm{dsDNA}$ conversion. However, it is not clear to what extent these secondary structures contribute to efficient sso function. The mechanisms involved in ss $\rightarrow$ dsDNA conversion are not well understood, although some features of the process have been defined. First, it seems that only host proteins are involved in the lagging-strand synthesis, most likely RNA polymerase, as shown in vivo for the ssoU of pUB110 (Boe et al., 1989), and in vitro for the sso $A$ of pT181 (Birch \& Khan, 1992). However, in the case of the lactococcal plasmid pWVO1 (an sso $A$ containing plasmid) this conversion has been proposed to be mediated by the host DNA primase (Leenhouts $e t$ al., 1991). Second, the amount of ssDNA molecules accumulated during RC-replication is plasmid- and host-dependent, indicating that the efficiency of this conversion depends upon the effectiveness with which the host machinery recognizes a given plasmid sso (Gruss et al., 1987; del Solar et al., 1987, 1993b; van der Lelie et al., 1989). However, it is not clear how or where the RNA primer synthesis initiates, and with which specific plasmid sequences it would pair to initiate the lagging-strand synthesis. To answer some of the above questions, we have started a systematic analysis of the regions of the streptococcal plasmid pMV158 involved in ss $\rightarrow$ dsDNA conversion. We show here that two plasmid elements belonging to $\operatorname{sso} A$ (namely, the recombination site $\mathrm{RS}_{\mathrm{B}}$ and a consensus 6 base sequence) contribute to the ss $\rightarrow$ dsDNA conversion in Streptococcus pneumoniae.

\section{METHODS}

Bacterial strains and plasmids. Strains employed for copy number determinations and stability assays were $S$. pneumoniae 708 (end-1 exo-2 trt-1 bex 4 malM594) and B. subtilis MB11 (lys-3 met $\mathrm{B}-10 \mathrm{bis} \mathrm{H}-2)$. Media and growth conditions were as described by Lacks et al.(1986). The plasmids employed, all harbouring the pMV158 replicon and conferring resistance to tetracycline, are listed in Table 1. Selective pressure for the antibiotic was kept at $1 \mu \mathrm{g} \mathrm{ml}^{-1}$ for $S$. pneumoniae and at $10 \mu \mathrm{g} \mathrm{ml}^{-1}$ for B. subtilis. In addition, plasmid pALTER and the Escherichia coli strains from a kit for site-directed mutagenesis (Promega) were used. Copy number determinations were performed as described by del Solar et al. (1987), with the aid of Computer DensitometerImageQuant equipment and software (Molecular Dynamics).

DNA preparation and manipulation. Purified plasmid DNAs were prepared from $S$. pneumoniae by two consecutive $\mathrm{CsCl} /$ ethidium bromide gradients as described by del Solar et al. (1987). Total DNA preparations (crude extracts) were made according to published procedures (Lacks et al., 1986). BAL31 deleted derivatives were constructed from plasmid pLS1 linearized at the single $A f I I$ site, treated as described by Puyet et al. (1988), and rescued by transformation of the pneumococcal host. The extent of the deletions was analysed by fine restriction mapping and by determination of the nucleotide sequence of the deletion junctions. Enzymes to manipulate the plasmid DNA were purchased from New England Biolabs or from Boehringer Mannheim, and used as specified by the vendors. All constructions were checked by determining the nucleotide sequence of the desired changes using the T7 Sequencing kit (Pharmacia).

Determination of the intracellular amounts of ssDNA. Analyses of ssDNA in cultures harbouring the plasmids of interest were made by electrophoresis on $1 \%(\mathrm{w} / \mathrm{v})$ agarose gels of total
DNA samples, followed by transfer to nitrocellulose filters with or without prior denaturation (te Riele et al., 1986). Filters were hybridized using ${ }^{32} \mathrm{P}$-labelled pLS1 DNA as a probe. When accumulation of ssDNA was measured in cultures treated with rifampicin and/or erythromycin, pneumococcal cultures harbouring the desired plasmids were grown to middle exponential phase (about $2 \times 10^{8}$ c.f.u. per $\mathrm{ml}$ culture). Then rifampicin (to $100 \mu \mathrm{g} \mathrm{ml}^{-1}$ ), erythromycin (to $100 \mu \mathrm{g} \mathrm{ml}^{-1}$ ) or both antibiotics were added. Culture samples were removed at various times and total DNA was analysed as described above. Direct quantification of the amount of ss- and dsDNA transferred to the filters, and calculation of the molecular ratios of ss/dsDNAs, were done with the aid of PhosphorImagerImageQuant equipment and software (Molecular Dynamics).

Site-directed mutagenesis. The 'Altered Sites' kit (Promega), designed for in vitro mutagenesis was used. To perform the mutagenesis, the 1243 bp EcoRI-Pst I fragment of pLS1 (coordinates 3170 and 5, respectively; see Fig. 1a) was cloned into pALTER digested with the same enzymes. The consensus sequence: $5^{\prime}$-TAGCGT-3' of the sso $A$ was changed into the sequence $5^{\prime}$-TATCGA- $3^{\prime}$ by the use of the oligonucleotide 5'-CGAGCCGAAAGGCTTATCGATTTCGGACGGACACGGA-3' (underlined letters indicate the mutation introduced within the wild-type sequence). This generates a new ClaI site $\left(5^{\prime}\right.$-ATCGAT-3'), which facilitated the screening of mutated plasmids. The desired mutant plasmid was isolated in E. coli, and the mutation was identified by digestion with ClaI. Then the cloned pLS1 DNA was inserted back into pLS1 by exchanging the EcoRI-Pst I fragment. The resulting plasmid (termed pLS1-CM) was rescued by transformation of $S$. pneumoniae, and the mutation was characterized by determination of its nucleotide sequence.

Database searching and structure prediction. Computer searches for homologies within the sequence of pMV158 were performed with the programs ALIGN and GAP (Dnastar, UK) with a window of 15 and a minimal homology of $55 \%$. Secondary structure predictions were done with the SQUIGGLES program (UWGCG, Wisconsin, USA).

\section{RESULTS AND DISCUSSION}

\section{Deletion mapping of ssoA}

The two sso regions of pMV158 are located on the plasmid coding strand. They are separated by the mob gene cassette, involved in plasmid conjugative mobilization (Fig. 1a). The $s s o U$ has been reported to be the preferred laggingstrand origin in B. subtilis (van der Lelie et al., 1989), but its efficiency in the ss $\rightarrow$ dsDNA conversion had not been analysed in the pneumococcal host. On the other hand, sso $A$ is efficiently recognized by $S$. pneumoniae, but not by B. subtilis (del Solar et al., 1987). We had experimentally defined the sso $A$ region as being between the HindIII and NcoI sites (pMV158 coordinates 4407-5349; Fig. 1a), the fragment removed during construction of plasmid pLS4 (Table 1). Deletion of this 942 bp region in pLS1, which already lacks $s s o U$, led to plasmids exhibiting an increased accumulation of ssDNA in $S$. pneumoniae, but not in $B$. subtilis (del Solar et al., 1987, 1993b). These plasmids, which lack the two sso regions, have a lower copy number, and a high segregational instability in both hosts, with a rate of loss of about $0 \cdot 1$ per cell per generation (del Solar et al., 1993b). We predicted that the sso $A$ should be included in a region with a high potential to generate 


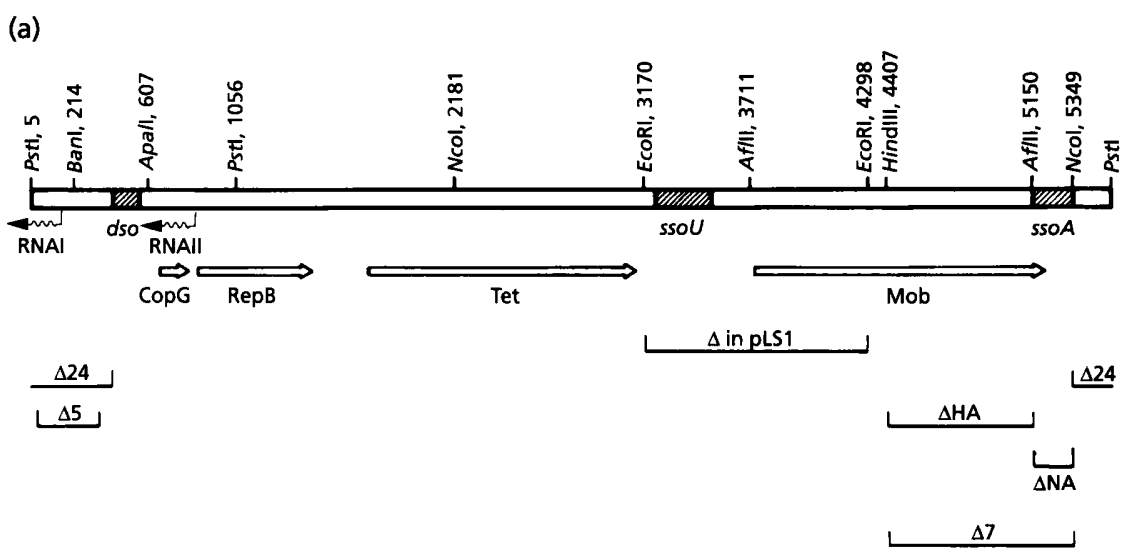

(c)

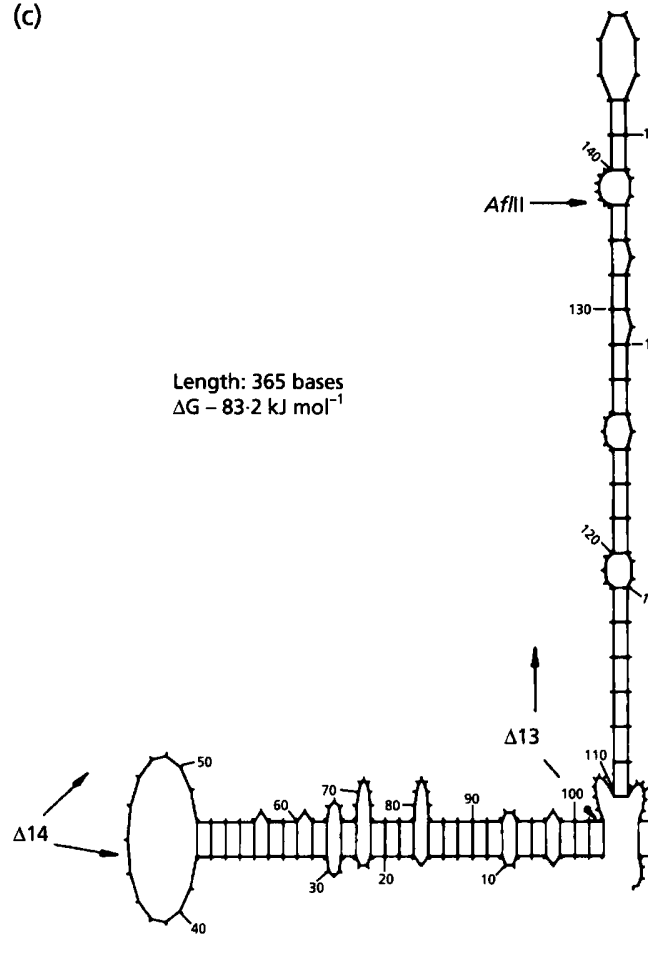

(b) $\quad 5016$

$\longrightarrow \Delta 14$

GTATAGACCGTCITAAAAGCGAATAAAGCGACTAAAAAACGAAAATICAATITTACGTCAG CATATCTGGCAGAATTITGCTITATTTCGCTGATITITTGCTTTTAAGTTAAAATGCAGTC

$\rightarrow \triangle 13$ 5077 CAAAATGACAAGATGCTAGGGAAATIAAGAGAGITAAIGCCTGATAAAGCCTITAAGAATIT GTTTACTGITCTACGATCCCTITAATTCTCTCAATTACGGACTATITCGGAATTCTTAAA

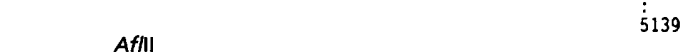

GITATCAGAACTTAAGgCGATTAAGCCAATCGTGAATATAATTAAAAAGGCTATTGAAAAGA CAATAGTCT GAATTCCGCTAATTCGGTTAGCACTTATATIAATTTTICCGATAACTTITC

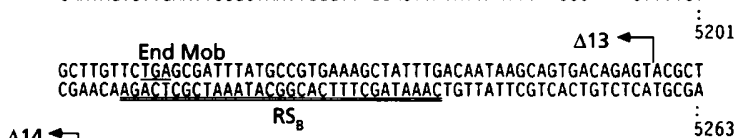

$\triangle 14$ $\mathrm{RS}_{\mathrm{B}}$ 5263

AGGACGTGCCGAGCCGAAAGGCTITIAGCGIIICGGACGGACACGGACAAAGGACGGCAGTCA TCCTGCACGGCTCGGCTITCCGAAATCGCAAAGCCTGCCTGTGCCIGTTICCIGCCGTCAGT

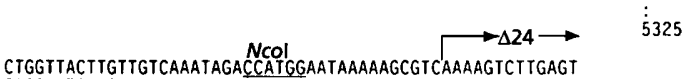
CTGGTTACTTGITGTCAAATAGACCATGGAATAAAAAGCGICAAAAGICTTGAGT
GACCAATGAACAACAGTTTATCTGGACCTTATTTTCGCAGTITCAGAACTCA $\vdots 380$

Fig. 1. Relevant features of pMV158. (a) Physical and genetic map of pMV158 and its deleted derivatives. The positions of the $d s o$ and the two sso are shadowed. Plasmid-encoded proteins, with direction of synthesis (arrowheads), are indicated. Wavy arrows indicate the position of the two antisense RNAs encoded by pMV158. Only relevant restriction sites are shown. (b) Nucleotide sequence around the ssoA. Coordinates of pMV158 are those by Priebe \& Lacks (1989). The recognition sites for $A$ f $/ I$ l and $N$ col are underlined. Locations of the recombination site $B$ ( $R S_{B}$; double underlined), and of the 6 bp consensus (bold) are shown. The borders of the $\Delta 13$ and $\Delta 14$ deletions and the left border of the $\Delta 24$ deletion are indicated. (c) Computer prediction of the secondary structure that could be generated from the sequence shown in (b). Features relevant for this work are indicated.

secondary structures, within which a nuclease-S1-sensitive site has been mapped (del Solar et al., 1987; Fig. 1c). This region is bordered by the restriction sites $A f I I I$ and NcoI (coordinates 5150 and 5349 of pMV158, respectively; Fig. 1b). Within this putative sso $A$-containing region, two conserved sequences have been proposed as being important elements of the lagging-strand origin of replication (Fig. 1c; Gruss et al., 1987; del Solar et al., 1987). The first of these is the recombination site, termed $\mathrm{RS}_{\mathrm{B}}$ (Novick et al., 1984), which is involved in plasmid recombination and generation of co-integrates among homologous plasmids. This region shares homologies with the pSC101 par locus, which is involved in stable maintenance and which includes a DNA-gyrase-binding site (Wahle \& Kornberg, 1988). The second of these, a 6 base consensus sequence $\left(5^{\prime}\right.$-TAGCGT- $\left.3^{\prime}\right)$, is located on the terminal loop of the putative hairpin within the sso $A$ of pLS1, pC221 and pC194 (del Solar et al., 1987; Fig. 1c). This consensus sequence is also present in the sso $A$ of several other RC-plasmids and coliphages, and has 
Table 1. Features of the plasmids used in this work

\begin{tabular}{|c|c|c|c|c|}
\hline Plasmid & $\begin{array}{l}\text { Size } \\
\text { (bp) }\end{array}$ & Mutation & $\begin{array}{l}\text { Relevant } \\
\text { features }\end{array}$ & $\begin{array}{l}\text { Reference/ } \\
\text { source }\end{array}$ \\
\hline pMV158 & 5536 & None & Wild-type & Burdett (1980) \\
\hline pLS1 & 4408 & $\Delta E c o$ RI-B fragment of pMV158 & $\Delta s s o U$ & Lacks et al. (1986) \\
\hline $\mathrm{pLS} 1 \Delta \mathrm{HA}$ & 3665 & $\Delta H i n \mathrm{dIII}-A f \operatorname{lI}$ of $\mathrm{pLS} 1$ & $\Delta s s o U$ & This work \\
\hline $\mathrm{pLS} 1 \Delta \mathrm{NA}$ & 4209 & $\begin{array}{l}\Delta A f I \mathrm{II}-\mathrm{NcoI} \text { small } \\
\text { fragment of pLS1 }\end{array}$ & $\Delta s s o A, \Delta s s o U$ & This work \\
\hline pLS1-CM & 4408 & $\begin{array}{c}5 \mathrm{bp} \text { change at the sso } A \\
\text { consensus in pLS1 }\end{array}$ & $\begin{array}{l}\Delta s s o U, \text { partially } \\
\text { affected in sso } A\end{array}$ & This work \\
\hline pLS1 113 & 4258 & $\begin{array}{l}\text { BAL31 deletion of } \mathrm{pLS} 1 \\
\text { linearized at } A f \mathrm{II}\end{array}$ & $\begin{array}{l}\Delta s s o U, \text { partial } \\
\text { deletion of } \\
\text { sso } A\end{array}$ & This work \\
\hline pLS1 114 & 4193 & $\begin{array}{l}\text { BAL31 deletion of pLS1 } \\
\text { linearized at } A f I \mathrm{II}\end{array}$ & $\begin{array}{l}\Delta s s o U, \text { partial } \\
\text { deletion of } \\
\text { sso } A\end{array}$ & This work \\
\hline pLS5 & 4076 & $\begin{array}{l}\text { in vivo spontaneous } \\
\text { deletion of } \mathrm{pLS} 1\end{array}$ & $\Delta s s o U, \Delta r n a I(\Delta 5)$ & $\begin{array}{l}\text { del Solar } \\
\text { et al. }(1987)\end{array}$ \\
\hline pLS4 & 3138 & $\begin{array}{l}\Delta 7: \text { HindIII-NcoI small } \\
\text { fragment of pLS5 }\end{array}$ & $\Delta s s o A, \Delta 5(\Delta r n a I)$ & $\begin{array}{l}\text { del Solar } \\
\text { et al. }(1987)\end{array}$ \\
\hline pLS4-EC & 4266 & $\begin{array}{l}\Delta 7, \text { EcoRI-B fragment of } \\
\text { pMV158 cloned in plS4 } \\
\text { (correct orientation) }\end{array}$ & $\Delta s s o A, \Delta 5(\Delta r n a I)$ & This work \\
\hline pLS4-EI & 4266 & $\begin{array}{l}\Delta 7, \text { EcoRI-B fragment of } \\
\text { pMV158 cloned in pLS4 } \\
\text { (incorrect orientation) }\end{array}$ & $\begin{array}{l}\Delta s s o A, \Delta 5(\Delta r n a I) \\
\text { non-functional } \\
\text { sso } U\end{array}$ & This work \\
\hline $\mathrm{pLS} 1 \Delta 24$ & 3838 & $\begin{array}{l}\text { BAL31 deletion of pLS1 } \\
\text { linearized at BanI }\end{array}$ & $\Delta s s o U, \Delta r n a I$ & $\begin{array}{l}\text { A gift from } P \text {. } \\
\text { Acebo }\end{array}$ \\
\hline
\end{tabular}

(a)

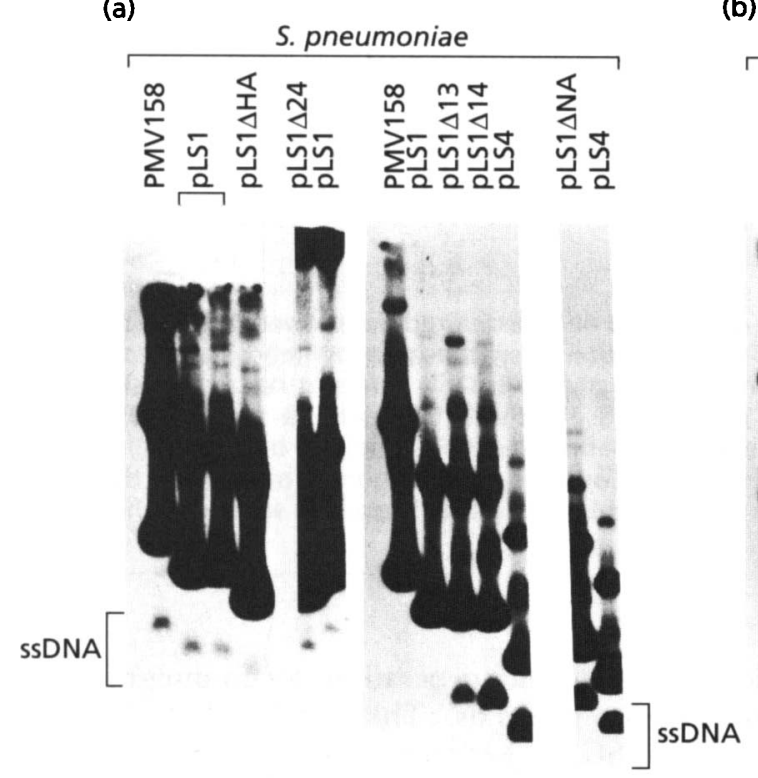

(b)

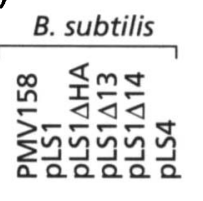

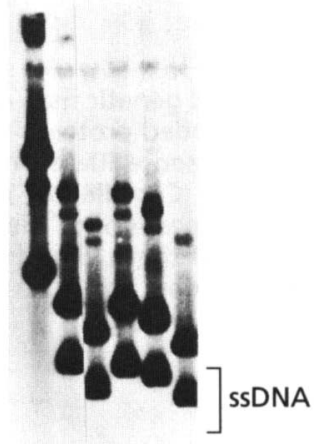

Fig. 2. Location of regions important for a functional ssoA. In addition to pMV158, plasmids derived from pLS1 ( $\Delta$ ssol $U$ ) were employed. The intracellular ssDNA accumulated by the indicated plasmid derivatives was detected by Southern blot hybridization of total DNA isolated from plasmid-containing cultures and electrophoresed in $1 \%$ agarose. DNA was transferred after denaturation, so that dsand SsDNA are visible. The positions of ssDNA in S. pneumoniae (a) and in B. subtilis (b) are bracketed. The leftmost lanes in part (a) are overexposed to visualize the ssDNA. been used as a criterion for the location of lagging-strand origins (Zaman et al., 1993). Part of the $\mathrm{RS}_{\mathrm{B}}$ as well as the consensus sequence are located within unpaired regions within the sso $A$ (Novick, 1989).
To define more precisely the boundaries of the sso $A$, AfIII-linearized pLS1 DNA was treated with BAL31 nuclease. Deleted pLS1-derivatives were rescued by transformation of $S$. pneumoniae, and several of them were 
Table 2. Copy number and molecular ratios of ss/dsDNA for plasmids bearing the pMV158 replicon

\begin{tabular}{|c|c|c|c|c|}
\hline \multirow[t]{2}{*}{ Plasmid } & \multicolumn{2}{|c|}{ S. pneumoniae } & \multicolumn{2}{|c|}{ B. subtilis } \\
\hline & $\begin{array}{c}\text { Copy } \\
\text { number* }\end{array}$ & $\begin{array}{l}\text { ss } / \text { ds DNA } \\
\text { ratio }\end{array}$ & $\begin{array}{c}\text { Copy } \\
\text { number }\end{array}$ & $\begin{array}{c}\text { ss } / \mathrm{ds} \text { DNA } \\
\text { ratio }\end{array}$ \\
\hline pMV158 & $36 \pm 6$ & $0.005 \pm 0.0006$ & $7 \pm 3$ & $<0.001$ \\
\hline pl.S1 & $22 \pm 2$ & $0.0065 \pm 0.0003$ & $4 \pm 1$ & $0 \cdot 67 \pm 0 \cdot 09$ \\
\hline pLS1 $1 \Delta \mathrm{HA}$ & $19 \pm 4$ & $0.0052 \pm 0 \cdot 0016$ & $4 \cdot 5 \pm 1$ & $1 \cdot 14 \pm 0 \cdot 10$ \\
\hline pJ.S1 13 & $12 \pm 4$ & $0 \cdot 10 \pm 0 \cdot 02$ & $4 \pm 1$ & $0.87 \pm 0.02$ \\
\hline $\mathrm{pl} S 1 \Delta 14$ & $10 \pm 3$ & $0 \cdot 20 \pm 0 \cdot(04$ & $4 \cdot 5 \pm 1$ & $0.75 \pm 0.07$ \\
\hline $\mathrm{pLS} 1 \Delta \mathrm{NA}$ & $15 \pm 3$ & $0 \cdot 31 \pm 0 \cdot 01$ & $\mathrm{ND}$ & ND \\
\hline pl.S4 & $11 \pm 3$ & $0 \cdot 34 \pm 0 \cdot 10$ & $3 \pm 1$ & $1 \cdot 02 \pm 0 \cdot 05$ \\
\hline pJ.S4-EC & $20 \pm 4$ & $0 \cdot 007 \pm 0 \cdot 001$ & $5 \pm 1$ & $<0 \cdot 001$ \\
\hline pIS4-EI & $12 \pm 4$ & $0 \cdot 10 \pm 0 \cdot 04$ & $5 \pm 1$ & $0 \cdot 84 \pm 0 \cdot 05$ \\
\hline pLS1-CM & $21 \pm 2$ & $0.017 \pm 0.004$ & $\mathrm{ND}$ & ND \\
\hline $\mathrm{pLS} 1 \triangle 24$ & $21 \pm 2$ & $0 \cdot 008 \pm 0.002$ & $\mathrm{ND}$ & ND \\
\hline pl.S5 & $46 \pm 3$ & $0.01 \pm 0.003$ & $9 \pm 1$ & $0.42 \pm 0 \cdot 02$ \\
\hline
\end{tabular}

ND, Not done.

* Measured as ds plasmid DNA.

characterized. Two plasmids were selected, both of them lacking the $\mathrm{RS}_{\mathrm{B}}$ but preserving the consensus sequence (pl.S1 $\Delta 13$ and pISS1 14 ; Fig. 1). Plasmids lacking the entire AflI-Ncol fragment (pLS1 $\Delta \mathrm{NA}$ ), or the HindIII - AfllI region (pLS1 $\Delta H A$ ) were also constructed (Table 1). As controls, pMV158 (wild-type), pLS1 $(\Delta s s o U)$, pLS1 $\Delta 24(\Delta s s o U)$ and pLS4 $(\Delta s s o U, \Delta s s o A)$ were used (Fig. 1a). All these plasmids were transferred to $S$. pneumoniae and to B. subtilis, and the ssDNA accumulated was detected (Fig. 2), and quantified (Table 2). In the pneumococcal host (Fig. 2a), no influence of the HindIII-AfII region in accumulation of ssDNA was observed, since the plasmid pLS1 $\triangle \mathrm{HA}$ accumulated as little ssDNA as pLS1 or pMV158. In addition, sequences located at $3^{\prime}$ of the $N$ col site (on the coding strand, Fig. 1b) do not play any role in the ss $\rightarrow \mathrm{dsDN} A$ conversion, since the amount of ssDNA detected for pLS1 $\Delta 24$ was similar to that of pLS1 (Fig. 2a). However, a 15-fold increase in the amount of $\$ S D N A$ was detected in plasmid pLS1 13 , with respect to pLS1, and a further twofold increase was found in pLS1 14 . Deletion of the whole $A f I I-N c o l$ region (pLS1 $\triangle \mathrm{NA}$ ) further increased the amount of ssDNA accumulated. These results demonstrate that the sso. A of plasmid pMV158 is located within the coordinates 5150 and 5349 , which experimentally narrows down this lagging-strand origin from 942 bp (del Solar et al., 1993b) to a 199 bp region (Fig. 1b). Regions similar in size have been defined for the sso $A$ of plasmid p'T181 (Birch \& Khan, 1992), and for the ssoU of pUB110 (Boe et al., 1989). In addition to this, we may also conclude that either the DNA sequence or its structure within the $\mathrm{RS}_{\mathrm{B}}$ plays an important role in the $\mathrm{ss} \rightarrow$ dsDNA conversion. However, the $\mathrm{RS}_{\mathrm{B}}$ is not essential since there is some $\operatorname{sso} A$-dependent conversion in its absence. This indicates that some other elements, in addition to the $\mathrm{RS}_{\mathrm{B}}$, must be involved in the $s s o A$ - (a)

(b)
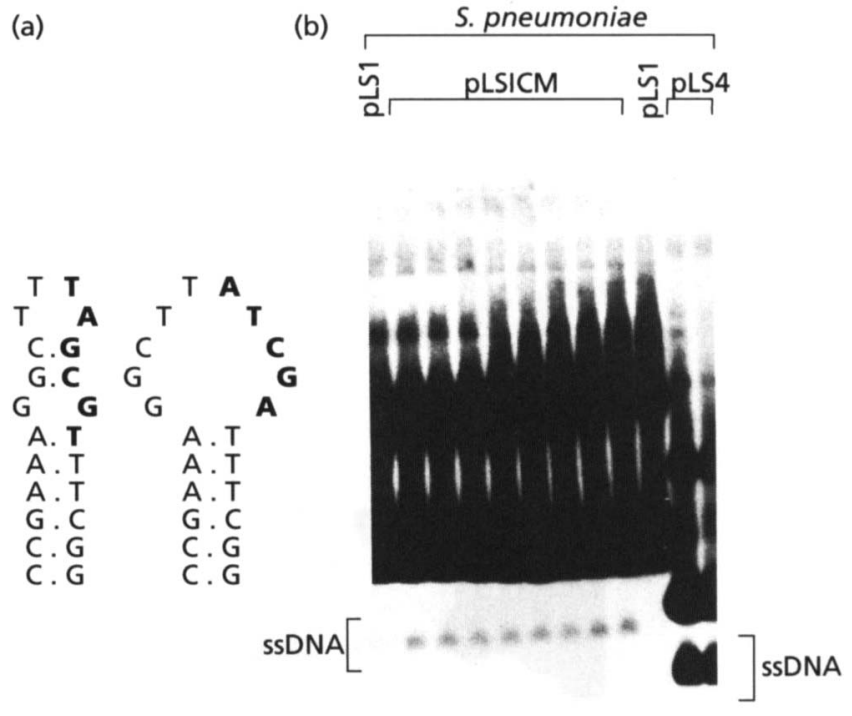

Fig. 3. Influence of the consensus sequence ( $5^{\prime}-$ TAGCGT- 3 ') on sso $A$ functionality. (a) Putative secondary structure generated on the loop of the ssoA in the wild-type pLS1 (left) and in the mutant pLS1-CM (right). Note that the mutation generates a consensus-like sequence 5'-TAtCGa-3'. (b) Accumulation of ssDNA in pneumococcal cultures harbouring the indicated plasmids. Samples were treated as in Fig. 2.

dependent conversion because pLS1 $\triangle \mathrm{NA}$ accumulated more ssDNA than pLS1 14 . In the case of $B$. subtilis, lack of functionality of the sso $A$ as a lagging-strand origin is demonstrated because none of the pLS1-deleted derivatives exhibited changes in the already high amount of ssDNA accumulated (Fig. 2b, Table 2).

\section{The consensus sequence of ssoA plays a role within pLS1 lagging-strand origin}

We had earlier postulated that the consensus $5^{\prime}$ TAGCGT-3' sequence, located on the loop of the secondary structure mapped in the sso $A$ region of pLS1, could play a role as part of this conversion signal (del Solar et al., 1987). To determine whether the consensus is involved in the ss $\rightarrow$ dsDNA conversion, we changed five bases of this sequence without significantly altering intrastrand pairing within the secondary structure (Fig. 3a). Due to the presence of a $\mathrm{T}$ just before the consensus, the mutation generated a weak consensus-like sequence 5'-TAtCGa-3' (Fig. 3a). Curiously, this sequence has only one mismatch (the central $\mathrm{T}$ ) as compared with the consensus found at the sso $A$ of plasmid pE194 (del Solar et al., 1987). Some of the features of the mutant plasmid, termed pI.S1-CM, were analysed only in $S$. pneumoniae because of the lack of functionality of $s$ so $A$ in $B$. subtilis. The phenotype of pLS1-CM was indistinguishable from its parental pLS1 with respect to copy number (Table 2). To evaluate statistically accumulation of ssDNA in cells harbouring pLS1-CM, eight independent DNA preparations were analysed. The results (Table 2, Fig. 3b) showed that a significant $2 \cdot 6$-fold increase in the amount of ssDNA was observed within cells harbouring PI.S1- 
(a)
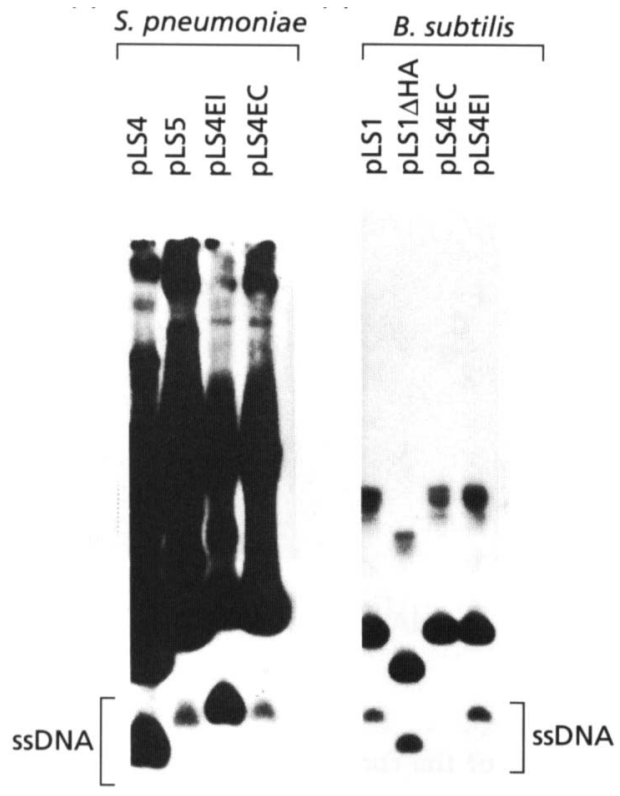

Fig. 4. The ssoU functionally replaces the $s s o A$ in $S$. pneumoniae. Cultures of $S$. pneumoniae or $B$. subtilis, harbouring the indicated plasmids, were treated as described in the legend to Fig. 2.

CM, as compared with pLS1 or pMV158. These results allowed us to conclude that the consensus sequence by itself may play a role within the conversion signal. However, we cannot rule out that the putative configuration of the loop of the sso $A$, which would be larger in the mutant than in the wild-type (Fig. 3a), could influence the ss $\rightarrow$ dsDNA conversion.

\section{ssoU is efficiently recognized by S. pneumoniae}

Pneumococcal cells harbouring pMV158 accumulate as small an amount of ssDNA as pLS1 (Fig. 2a), which could be due solely to the presence of the functional sso $A$. To test whether sso $A$ could be replaced by the sso $U$ in $S$. pneumoniae, the 1128 bp EcoRI fragment B of pMV158, which is removed in pLS1 and its derivatives (Fig. 1a), was cloned into the single EcoRI site of plasmid pLS4 in the two possible orientations (Table 1). If the ssoU acts as an efficient lagging-strand origin in pneumococci, low amounts of ssDNA should be detected only when the EcoRI-B fragment is cloned in the correct (pLS4-EC), but not in the opposite (pLS4-EI), orientation. This was indeed the case, and pLS4-EC accumulated little ssDNA, whereas pLS4-EI behaved similarly to its parental pLS4 (Fig. 4a). In addition, the decrease in copy number (measured as dsDNA) observed for pLS4 was restored to the levels of pLS1 only in the case of pLS4-EC (Table 2). Similar results on ssDNA accumulation were found for $B$. subtilis (Fig. 4b), although the number of copies of the plasmids did not vary (Table 2). This finding supports previous observations indicating that the sso $U$ is the functional lagging-strand origin in B. subtilis (van der
Lelie et al., 1989). We can thus conclude that the pMV158sso $U$ acts as an efficient conversion signal in both hosts. It remains to be shown whether the two sso present in pMV158 play different roles in $S$. pneumoniae. Due to its location within the plasmid (Fig. 1a), we can speculate that the sso $U$ would be the lagging-strand origin preferentially used after conjugative plasmid transfer, since deletion of a region which encompasses the sso $U$ resulted in loss of the mobilization function (Priebe \& Lacks, 1989). In this sense, it is curious that the two sso regions are placed just before and just after the mob gene. Alternatively, RC-replication from the dso would lead to the exposure of ssoU as an ssDNA region before the replisome reaches the $s s o A$. If the host machinery follows a 'first-exposed, first-used' pattern for $s s \rightarrow$ dsDNA conversion, then sso $U$ should be the preferential laggingstrand origin employed in pMV158.

\section{Pneumococcal RNA polymerase is involved in the ss $\rightarrow$ dsDNA conversion}

RC-plasmids pUB110 and p'T181 require the host RNA polymerase for ss $\rightarrow$ dsDNA conversion (Boe et al., 1989; Birch et al., 1992). To determine whether pMV158 uses the $S$. pneumoniae RNA polymerase for lagging-strand synthesis, exponentially growing cells harbouring pLS1 $(\Delta s s o U)$, pLS4-EC $(\Delta s s o A)$ or pLS4 $(\Delta s s o U \Delta s s o A)$ were subjected to inhibition of RNA and/or protein synthesis, by treatment with rifampicin, erythromycin or both antibiotics simultaneously. Rifampicin treatment would prevent RNA polymerase activity, whereas inhibition of protein synthesis would hinder synthesis of the initiation of replication $\mathrm{RepB}$ protein, thus preventing (at least partially) further rounds of replication from the plasmid dso. The amount of plasmid DNA was quantified and the molecular ratio of ss/dsDNA was plotted as a function of the time of treatment (Fig. 5). A 10-fold increase in this ratio was observed in the cultures in which RNA or RNA and protein synthesis were inhibited, but only if they harboured plasmids having one or another sso (Fig. 5a, b). A net increase in the amount of ssDNA accumulated upon inhibition of RNA polymerase could be observed in either pLS1 and pLS4-EC, but not in pLS4 (Fig. 6). No such accumulation was found for the sso-containing plasmids in the absence of antibiotics (Fig. 6). Consequently, the accumulation of ssDNA after inhibition of RNA synthesis (but not of protein synthesis) suggests that an active RNA polymerase is needed to avoid accumulation of ssDNA within the cells. This in turn indicates that the pneumococcal RNA polymerase is the enzyme involved in priming DNA synthesis from both lagging-strand origins. These results are supported by the observation that no significant changes were observed for the sso-deleted plasmid pLS4 (Fig. 5c). We conclude that the same host enzyme is involved in synthesis of a primer in two plasmid regions located far apart, and apparently performing the same function. The sso $A$ and the sso $U$ regions exhibit high potential to generate secondary structures (data not shown) and, since both sso regions are recognized by the host RNA polymerase, we expected to find some sequence conservation among them. However, computer searches 


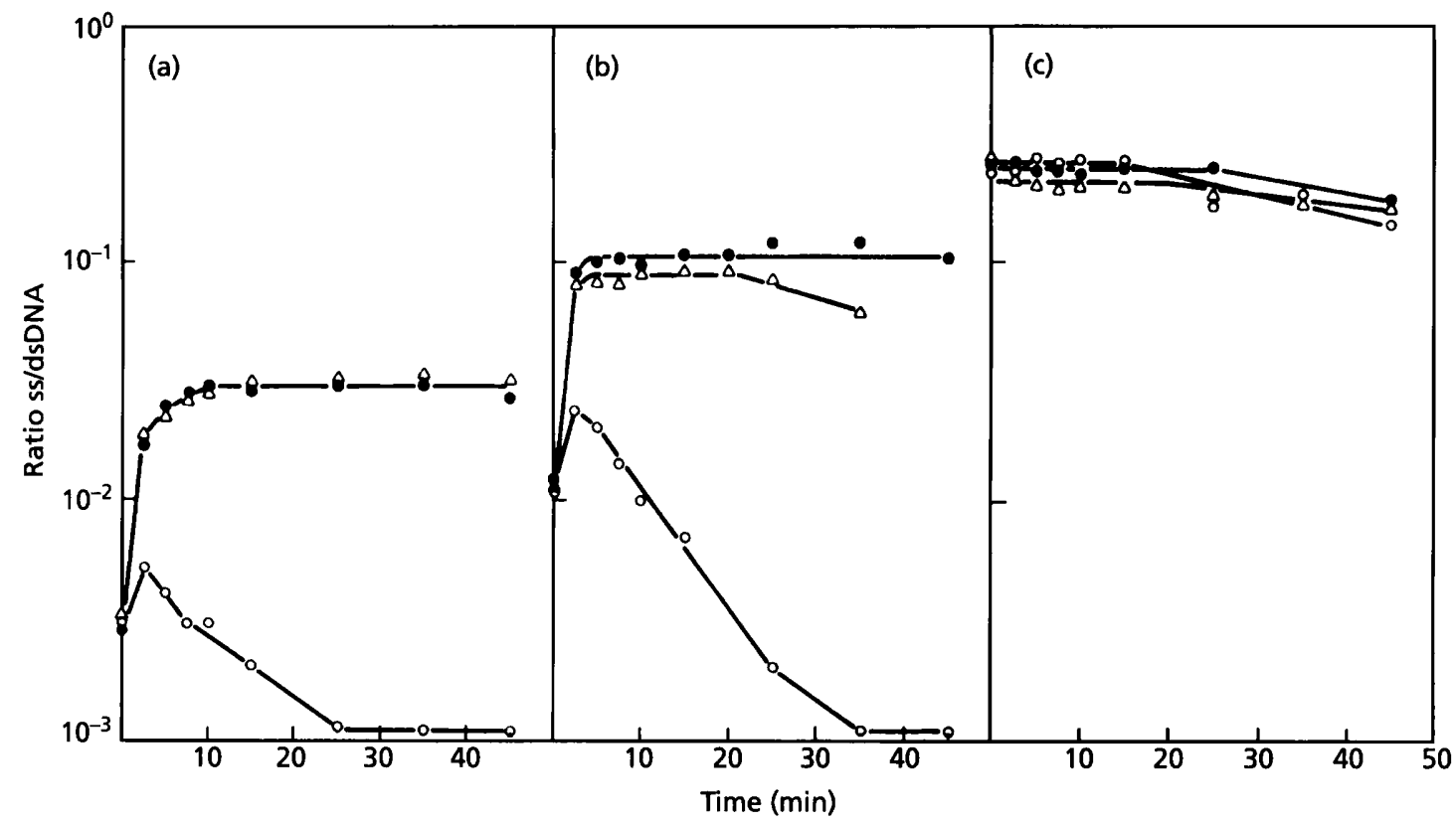

Fig. 5. Influence of rifampicin on ss $\rightarrow$ dsDNA conversion. Pneumococcal cultures harbouring pLS1 [ $\Delta$ ssoU, panel (a)], pLS4-EC [ $\Delta$ ssoA, panel (b)] or pLS4 [ $\triangle$ ssoA $\Delta s s o U$, panel (c)] were treated with rifampicin $(\triangle)$, erythromycin $(O)$ or both antibiotics (O). At the indicated times, samples were removed, and the molecular ratios of ss/dsDNA accumulated were quantified.

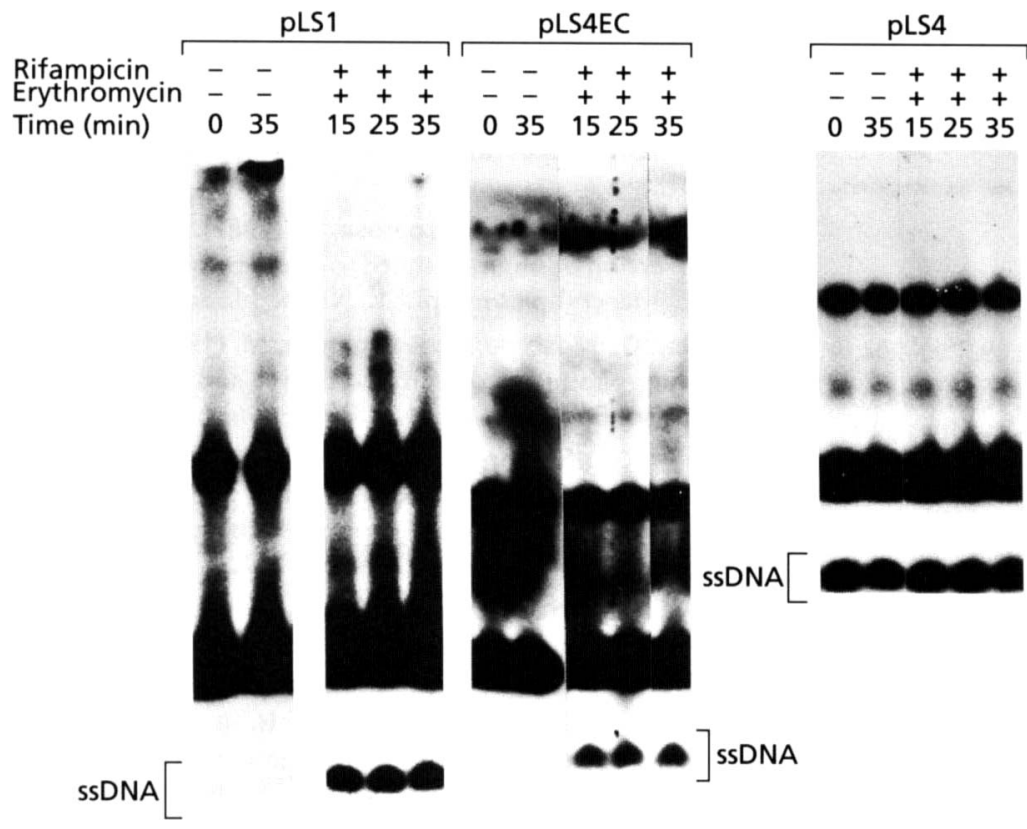

Fig. 6. Intracellular accumulation of ssDNA. Pneumococcal cultures harbouring the indicated plasmids were treated $(+)$ or not treated $(-)$ with rifampicin or erythromycin as in Fig. 5. Samples were removed at various times, and total DNA was analysed as in Fig. 2. The position of ssDNA is indicated.

showed neither significant homologies between the ssoU and the $\operatorname{sso} A$, nor any putative promoter-like sequence (data not shown). Plasmids having a low $\mathrm{G}+\mathrm{C}$ content, like pMV158, do have several sequences identical to the promoter -10 consensus region, but their significance is questionable. Consequently, which DNA signals are recognized by the RNA polymerase is unknown.
An interesting finding is that, in spite of the high amount of ssDNA accumulated in cells harbouring plasmids devoid of sso, those plasmids could be maintained under selective pressure. This indicates that at least an alternative pathway for the conversion of ss $\rightarrow$ dsDNA should exist. How then could plasmids with defective lagging-strand origins replicate? Based upon indirect evidence, we 
proposed that an alternative sso could exist within the tet gene of pMV158 (del Solar et al., 1987). If this alternative sso is functional, a host function, other than RNA polymerase, and insensitive to inhibition of protein synthesis, should be involved in this weak mechanism of ss $\rightarrow$ dsDNA conversion. Based on our results, we believe that both sequence-specific elements $\left(\mathrm{RS}_{\mathrm{B}}\right.$ and 6-mer consensus) and secondary structures could be the best combination for a region to function as an sso. The results obtained so far with pMV158 allow us to draw the following sequence of events in the ss $\rightarrow$ dsDNA conversion process. Lagging-strand synthesis would initiate from an ssDNA plasmid template which should be covered by the host single-stranded DNA binding protein, except in those regions having intrastrand pairing. Secondary structures, in conjunction with paired and unpaired sequence-specific sites, could act as signals which would be recognized by the host RNA polymerase. Upon synthesis of an RNA primer, DNA polymerase III would proceed to synthesize DNA until reaching an sso which would now be a DNA-RNA hybrid. The last stage would be the removal of the RNA and termination of DNA synthesis, which is performed by the host DNA polymerase I (Díaz et al., 1994).

\section{ACKNOWLEDGEMENTS}

Thanks are due to M. T. Alda, P. Valiente and R. Galán for technical assistance. Corrections to the English by D. M. Main are very much appreciated. Research was financed by CICYT (Grant BIO94-1029), and Comunidad Autónoma de Madrid (Grant 190/92). M.G.K. is the recipient of a Celestino Mutis fellowship from the Agencia Española de Cooperación Internacional.

\section{REFERENCES}

Birch, P. \& Khan, S. A. (1992). Replication of single-stranded plasmid pT181 DNA in vitro. Proc Natl Acad Sci US A 89, 290-294.

Boe, L., Gros, M. F., te Riele, H., Ehrlich, S. D. \& Gruss, A. D. (1989). Replication origins of single-stranded DNA plasmid pUB110. $J$ Bacteriol 171, 3366-3372.

Bron, S., Luxen, E. \& Swart, P. (1988). Instability of recombinant pUB110 plasmids in Bacillus subtilis: plasmid-encoded stability function and effects of DNA inserts. Plasmid 19, 231-241.

Díaz, A., Lacks, S. A. \& López, P. (1994). Multiple roles for DNA polymerase $I$ in establishment and replication of the promiscuous plasmid pLS1. Mol Microbiol 14, 773-783.

Gigliani, F., Ciotta, C., del Grosso, M. F. \& Battaglia, P. (1993). pR plasmid replication provides evidences that single-stranded DNA induces the SOS system in vivo. Mol \& Gen Genet 238, 333-338.

Gruss, A. D., Ross, H. F. \& Novick, R. P. (1987). Functional analysis of a palindromic sequence required for normal replication of several staphylococcal plasmids. Proc Natl Acad Sci US A 84, 2165-2169.
Gruss, A. D. \& Ehrlich, S. D. (1989). The family of highly interrelated single-stranded deoxyribonucleic acid plasmids. Microbiol Rev 53, 231-241.

Lacks, S. A., López, P., Geenberg, B. \& Espinosa, M. (1986). Identification and analysis of genes for tetracycline resistance and replication functions in the broad-host-range plasmid pLS1. $J$ Mol Biol 192, 753-765.

Leenhouts, K. J., Tolner, B., Bron, S., Kok, J., Venema, G. \& Seegers, J. F. M. L. (1991). Nucleotide sequence and characterization of the broad-host-range lactococcal plasmid pWV01. Plasmid 26, 55-66.

van der Lelie, D., Bron, S., Venema, G. \& Oskam, L. (1989). Similarity of minus origins of replication and flanking open reading frames of plasmids pUB110, pTB913 and pMV158. Nucleic Acids Res 17, 7283-7294.

McKenzie, T., Hoshino, T., Tanaka, T. \& Sueoka, N. (1986). The nucleotide sequence of pUB110: some salient features in relation to replication and its regulation. Plasmid 15, 93-103.

Novick, R. P. (1989). Staphylococcal plasmids and their replication. Annu Rev Microbiol 43, 537-565.

Novick, R. P., Projan, S. J., Rosenblum, W. \& Edelman, I. (1984). Staphylococcal plasmid cointegrates are formed by host- and phage-mediated general $\mathrm{rec}$ systems that act on short regions of homology. Mol \& Gen Genet 195, 374-377.

Priebe, S. D. \& Lacks, S. A. (1989). Region of the streptococcal plasmid pMV158 required for conjugative mobilization. J Bacteriol 171, 4778-4784.

Projan, S. J. \& Novick, R. P. (1988). Comparative analysis of five related staphylococcal plasmids. Plasmid 19, 203-221.

Puyet, A., del Solar, G. \& Espinosa, M. (1988). Identification of the origin and direction of replication of the broad-host-range plasmid pLS1. Nucleic Acids Res 16, 115-133.

te Riele, H., Michel, B. \& Ehrlich, S. D. (1986). Are single-stranded circles intermediates in plasmid DNA replication? EMBO J $\mathbf{5}$, 631-637.

del Solar, G., Puyet, A. \& Espinosa, M. (1987). Initiation signals for the conversion of single stranded to double stranded DNA forms in the streptococcal plasmid pLS1. Nucleic Acids Res 15, 5561-5580.

del Solar, G., Moscoso, M. \& Espinosa, M. (1993a). Rolling circlereplicating plasmids from Gram-positive and Gram-negative bacteria : a wall falls. Mol Microbiol 8, 789-796.

del Solar, G., Kramer, G., Ballester, S. \& Espinosa, M. (1993b). Replication of the promiscuous plasmid pLS1: a region encompassing the minus origin of replication is associated with stable plasmid inheritance. Mol \& Gen Genet 241, 97-105.

Wahle, E. \& Kornberg, A. (1988). The partition locus of plasmid pSC101 is a specific binding site for DNA gyrase. EMBO J 7, 1889-1895.

Zaman, S., Radnedge, L., Richards, H. \& Ward, J. M. (1993). Analysis of the site for second-strand initiation during replication of the Streptomyces plasmid pIJ101. J Gen Microbiol 139, 669-676.

Received 16 September 1994; revised 25 October 1994; accepted 28 October 1994. 